\title{
Serum biomarker release patterns following alcohol septal ablation for treatment of hypertrophic cardiomyopathy
}

This article was published in the following Dove Press journal:

Current Biomarker Findings

12 December 2014

Number of times this article has been viewed

\author{
Joseph D Foley' \\ Craig S Miller ${ }^{2}$ \\ J Darrell Sneed' \\ Jeffrey L Ebersole ${ }^{2}$ \\ Richard J Kryscio ${ }^{3}$ \\ John T McDevitt ${ }^{4}$ \\ Charles L Campbell ${ }^{5}$
}

'Gill Heart Institute and Division of Cardiovascular Medicine, Department of Internal Medicine, ${ }^{2}$ Department of Oral Health Practice, College of Dentistry, ${ }^{3}$ Department of Statistics, University of Kentucky, Lexington, KY, ${ }^{4}$ Departments of Bioengineering and Chemistry, Rice University, Houston, TX, USA; ${ }^{5}$ Division of Cardiololgy, University of Tennessee at Chattanooga, Erlanger Health Systems, TN, USA
Correspondence: Charles L Campbell University of Tennessee Chattanooga, Erlanger Health Systems,

979 East Third Street,

Suite C-520, Medical Mall,

Chattanooga TN, 37403, USA

Tel + | 423778566 |

Fax +I 4237785664

Email charles.campbell@erlanger.org
Abstract: Alcohol septal ablation (ASA) is employed to relieve the pressure gradient associated with symptomatic hypertrophic cardiomyopathy. Serum concentrations of cardiac troponin I, creatine kinase MB band, brain natriuretic protein, matrix metalloproteinase-9, myoglobin, C-reactive protein, tumor necrosis factor-alpha, soluble CD40 ligand, interleukin-6, adiponectin, interleukin-1 $\beta$, myeloperoxidase, and soluble intercellular adhesion molecule-1 were determined at baseline and at $8,16,24$, and 48 hours in patients with hypertrophic cardiomyopathy presenting for ASA. Comparisons were made with 107 healthy control subjects. Sixteen hours following ASA, serum levels rose over 800-fold for cardiac troponin I, 70-fold for creatine kinase MB band, and 11-fold for myoglobin $(P<0.001)$. C-reactive protein and interleukin-6 both rose slowly and became significantly elevated at 16 and 48 hours, respectively. Matrix metalloprotease- 9 rapidly increased two-fold at 8 hours, but returned to baseline thereafter. Other biomarkers evaluated either trended downward or showed little change from baseline. Among the ASA patients, baseline serum concentration of all biomarkers, except for matrix metalloproteinase-9, soluble intercellular adhesion molecule-1, and myeloperoxidase, were elevated in the ASA group compared with the controls. These findings suggest that hypertrophic cardiomyopathy is a proinflammatory and prothrombotic state. The time-dependent changes in these biomarkers suggest they may be useful in predicting the success of ASA and could potentially offer insight into biochemical events during the time course of acute myocardial ischemia.

Keywords: alcohol septal ablation, cardiac biomarkers, hypertrophic cardiomyopathy, inflammation, acute myocardial infarction

\section{Introduction}

Hypertrophic cardiomyopathy (HCM) is a genetic disorder characterized by hypertrophy of the left ventricle in the absence of dilation or another disease process that could produce hypertrophy. ${ }^{1}$ The histopathological features of the affected myocardium in HCM include disorganized myocytes and myocardial fibrosis. Dyspnea and other symptoms of heart failure are common among HCM patients and are related in part to the presence of an intracardiac pressure gradient. Invasive procedures such as surgical myomectomy or alcohol septal ablation (ASA) are considered to relieve the gradient when symptoms are refractory to medical management. ASA involves controlled myocardial necrosis of the proximal interventricular septum via introduction of ethanol into the first septal perforating branch of the left anterior descending coronary artery. ${ }^{2-4}$ The resulting infarction is typically transmural, involving approximately $8 \%$ of the left ventricle located inferior in the basal septum. ${ }^{5}$ An evaluation of biomarker release among patients undergoing ASA demonstrated that serum levels of cardiac troponin I (TnI), 
creatine kinase, and creatine kinase $\mathrm{MB}$ band (CK-MB) but not B-type natriuretic peptide (BNP) levels, correlated with the size of the ASA-induced infarct. ${ }^{6}$

TnI and troponin T are the most specific cardiac biomarkers for detection of myocardial necrosis and have become the biomarkers of choice for identification of an acute myocardial infarction (AMI). ${ }^{7,8}$ Additionally, serial measurements of troponin levels and other cytoplasmic enzymes, such as creatine kinase, CK-MB, and lactate dehydrogenase, are used to estimate infarct size in AMI. As noted, serum levels of TnI, creatine kinase, and CK-MB have also been used to determine infarct size in patients undergoing ASA. ${ }^{6,9,10}$ Matrix metalloproteinase (MMP)-9 and BNP have also been studied during the first 48 hours post-ASA. ${ }^{11,12}$ ASA represents a clinical model of AMI in humans that has been employed to follow the precise kinetic analysis of biomarker release following induction of ischemia and infarction. ${ }^{13}$ In contrast with other models, such as percutaneous coronary intervention, the presence of active coronary disease is unlikely in HCM patients undergoing ASA and patients can serve as their own controls. ${ }^{14}$

In this study, we extend previous studies in this field by comparing baseline levels of a variety of serum biomarkers known to be associated with myocardial necrosis, systemic inflammation, and thrombosis between a group of patients with HCM and healthy controls. We quantified the serum concentrations of these biomarkers serially during the 48 hours following ASA. Our goals were to further characterize the inflammatory state that accompanies HCM and to correlate biomarker release with ASA-induced myocardial injury and the subsequent reduction in left ventricular outflow tract gradient.

\section{Materials and methods Patient selection}

Patients were eligible for study participation if they underwent ASA as treatment for HCM at the University of Kentucky Chandler Medical Center between May 1, 2007 and May 31, 2011. This study population has been previously reported with respect to the comparative salivary fluid changes that occur before, following ASA, and percutaneous coronary intervention. ${ }^{15,16}$ Diagnosis of obstructive HCM was based on established clinical and echocardiographic criteria. At this institution, ASA is performed for patients with HCM if they have severe symptoms (dyspnea, New York Heart Association heart failure class III to IV, Canadian Cardiovascular Society angina class III to IV, or recurrent exercise-induced syncope), and are refractory to medical therapy with left ventricular outflow tract gradients of $\geq 30 \mathrm{mmHg}$ at rest or $\geq 50 \mathrm{mmHg}$ with provocation. Exclusion criteria included age younger than 18 years, unable or unwilling to provide informed consent or provide samples, recently treated with chemotherapeutic drugs, anti-organ rejection drugs, or significant immune modulators within the last 3 months (or during the course of the study), febrile illness or active infection at the time of enrollment, or pregnancy. Once screened, 21 patients were treated with echocardiographyguided ASA and were enrolled in the study. In addition, a cohort of healthy subjects (ie, with a medical history negative for cardiovascular disease and cardiac symptoms) were recruited $(n=107)$ to provide baseline comparative samples for this study. All patients provided informed consent for their procedure, after explaining the risks and benefits of their participation. The study was approved by the institutional review board at the University of Kentucky. All study participants were provided with financial remuneration for their participation.

\section{Catheter ablation}

Femoral venous and arterial access was obtained in all patients. Catheters were positioned under fluoroscopic guidance. If necessary, a temporary pacemaker electrode was positioned in the right ventricle for transvenous pacing. Coronary arteriography was performed using diagnostic catheters with hand-injected contrast in multiple cineographic projections. Anticoagulation was obtained with intra-arterial bolus doses of heparin in order to maintain a therapeutic activated clotting time. Antiplatelet therapy was achieved with oral aspirin prior to the procedure. To assess for the presence and subsequent absence of left ventricular outflow tract pressure gradients, simultaneous pressure recordings between the left ventricle and aorta were measured. For this, a pigtail catheter was positioned in the ascending aorta and a trans-septal puncture was made in the interatrial septum after obtaining right femoral venous access. Following this, an 8 French Berman angiographic catheter was advanced across the septum, through the left atrium, and into the left ventricular cavity. For the ablation, a coronary guide catheter was positioned in the ostium of the left main coronary artery, and a 0.014 -inch Whisper guidewire and a $2.0 \mathrm{~mm}$ by $8 \mathrm{~mm}$ balloon were used to selectively engage the first septal perforating arteries of the left anterior descending coronary artery. To confirm the distribution of each septal perforating artery, the balloon was inflated at the origin of the septal branch and radiographic contrast was injected through the lumen of the balloon to exclude retrograde leakage of injected fluid into 
the left anterior descending artery and to exclude the presence of a large collateral to one of the other epicardial coronary arteries. Immediately following this, Definity ${ }^{\circledR}$ contrast was injected through the balloon lumen during simultaneous transthoracic echocardiography to assess the localization and extent of the myocardial territory perfused by the target septal perforator. If no leakage occurred and the perfused myocardial segment was considered responsible for the occurrence of the left ventricular outflow tract gradient, approximately $1 \mathrm{cc}$ of $96 \%$ ethanol was slowly injected through the balloon lumen for each vessel that was ablated. The balloon was left inflated for 5 minutes after infusion of alcohol to ensure ablation was complete. This procedure was repeated in the same, or additional septal perforators until the left ventricular outflow tract gradient decreased to $<25 \mathrm{mmHg}$ (which was our definition of procedural success), and ideally $0 \mathrm{mmHg}$. After the procedure, patients were monitored for 72 hours to observe for potential adverse events. If complete heart block occurred, the patient was temporarily paced transvenously until a pacing implantable cardioverter-defibrillator could be implanted. Follow-up occurred in our cardiology clinic within 2-4 weeks after discharge, at which time clinical symptoms were reassessed and repeat transthoracic echocardiography was performed.

\section{Serum specimens}

Serum samples were collected by venipuncture from each subject at baseline and at $8,16,24$, and 48 hours after the procedure. Patients undergoing ASA were fasting at the time of the first serum collection, those who constitute the control sample were not. The ASA procedures tended to occur in the morning. Samples were transported to the local laboratory on ice, centrifuged, separated into aliquots, and stored at $-80^{\circ} \mathrm{C}$ until analyzed. All samples were analyzed in duplicate within 3 months of storage. Serum levels of the classic cardiac biomarkers, ie, cardiac TnI, CK-MB, myoglobin, and BNP, were analyzed using a Beckman Access in the hospital's Clinical Laboratory Improvement Amendments-certified laboratory. Concentrations of serum C-reactive protein, tumor necrosis factor-alpha (TNF- $\alpha$ ), soluble CD40 ligand (sCD40L), interleukin (IL)-6, adiponectin, MMP-9, IL-1 $\beta$, myeloperoxidase, and soluble intercellular adhesion molecule 1 (sICAM-1) were determined in duplicate using Luminex IS100-based multiplex kits (Millipore, St Charles, MO, USA) according to the manufacturer's directions in the University of Kentucky Center for Oral Health Research laboratory. Standards were included on all runs and all results are reported within the linearity of the assays.

\section{Statistical analysis}

All statistical analyses were performed using PC SAS version 9.2 software (SAS Institute Inc., Cary, NC, USA) with significance determined at the $P<0.05$ level. Continuous demographic variables were presented as the mean and standard deviation and discrete demographic variables as frequencies and percentages. The biomarker levels, both raw-scale and log-transformed, were presented as the mean and standard deviation by visit time. Compared with baseline biomarker values, the relative fold change was calculated and graphed for each biomarker at each time interval. Biomarker levels were compared between each time point versus control using the Wilcoxon two-sample test, and levels were also compared between each follow-up time point and baseline using the Wilcoxon signed rank sum test.

\section{Results}

A total of 21 patients were consented, and 19 underwent ASA for treatment of symptomatic HCM. All were Caucasian, the majority were women (63.2\%), and the mean age was 58.6 years. Baseline characteristics, including cardiovascular drug usage, are presented in Table 1. Clinical characteristics preprocedure indicated New York Heart Association class III or higher heart failure symptoms in $28 \%$, syncope or presyncope in 33\%, and Canadian Cardiovascular Society class III or higher angina in $22 \%$ of patients.

Echocardiographic data both preprocedure and postprocedure indicate that $94 \%$ had a left ventricular ejection fraction $>55 \%$ (Table 2). Baseline interventricular septal dimension by two-dimensional M-mode was $16.2 \mathrm{~mm}$ preprocedure and $15 \mathrm{~mm}$ postprocedure. Left atrial size was reduced

Table I Demographics of alcohol septal ablation patients

\begin{tabular}{|c|c|}
\hline Mean age, years & 58.6 \\
\hline Sex & Female $63.2 \%$, male $36.8 \%$ \\
\hline Ethnicity & Caucasian $100 \%$ \\
\hline Atrial fibrillation & $11 \%$ \\
\hline Chronic obstructive pulmonary disease & $11 \%$ \\
\hline Coronary artery disease & $11 \%$ \\
\hline Diabetes mellitus & $11 \%$ \\
\hline Family history of sudden cardiac death & $22 \%$ \\
\hline Hypertension & $61 \%$ \\
\hline Hyperlipidemia & $56 \%$ \\
\hline Peripheral vascular disease & $58.6 \pm 13.4$ years (mean, SD) \\
\hline Prior stroke & Female $63.2 \%$, male $36.8 \%$ \\
\hline Tobacco abuse & Caucasian $100 \%$ \\
\hline Baseline creatinine & $11 \% \pm 32.3 \%$ (mean, SD) \\
\hline Beta-blocker usage & $11 \% \pm 32.3 \%$ (mean, SD) \\
\hline Calcium channel blocker usage & $11 \% \pm 32.3 \%$ (mean, SD) \\
\hline Antiarrhythmic drug usage & II\% $\% 32.3 \%$ (mean, SD) \\
\hline
\end{tabular}

Abbreviation: SD, standard deviation. 
Table 2 Echocardiographic data before and after ablation

\begin{tabular}{lll}
\hline & $\begin{array}{l}\text { Before } \\
\text { ablation }\end{array}$ & $\begin{array}{l}\text { After } \\
\text { ablation }\end{array}$ \\
\hline LV ejection fraction $>55 \%$ & $94 \%$ & $94 \%$ \\
LV end-diastolic dimension & $45.4 \mathrm{~mm}$ & $49.4 \mathrm{~mm}$ \\
LV end-systolic dimension & $26.8 \mathrm{~mm}$ & $27.0 \mathrm{~mm}$ \\
Left atrial size & $49.9 \mathrm{~mm}$ & $44.3 \mathrm{~mm}$ \\
Interventricular septal diameter & $16.2 \mathrm{~mm}$ & $15.0 \mathrm{~mm}$ \\
LV posterior wall diameter & $13.8 \mathrm{~mm}$ & $13.5 \mathrm{~mm}$ \\
Right ventricular systolic pressure & $23 \mathrm{mmHg}$ & $31.6 \mathrm{mmHg}$ \\
Mitral regurgitation grade & 1.5 & 1.0 \\
Tricuspid regurgitation grade & 0.7 & 0.5 \\
Resting LV outflow tract gradient & $69.3 \mathrm{mmHg}$ & $15.1 \mathrm{mmHg}$ \\
Systolic anterior motion of mitral valve & $100 \%$ & $30 \%$ \\
\hline
\end{tabular}

Abbreviation: LV, left ventricular.

from $49.9 \mathrm{~mm}$ preprocedure to $44.2 \mathrm{~mm}$ postprocedure. Dynamic systolic anterior motion of the mitral valve improved from $100 \%$ preprocedure to $30 \%$ postprocedure, with associated improvement in grade of mitral regurgitation from +1.5 to +1 . Resting left ventricular outflow tract gradients improved from $69.3 \mathrm{mmHg}$ preprocedure to 15.1 $\mathrm{mmHg}$ at postprocedure follow-up.

On average, 1.83 septal arteries were ablated per patient, with a mean of $0.98 \mathrm{cc}$ ethanol per vessel. Intraprocedural success, as defined by postprocedure reduction in left ventricular outflow tract gradients to $<25 \mathrm{mmHg}$, was accomplished in $83 \%$ of subjects with a mean immediate improvement in left ventricular outflow tract gradient from $94.6 \mathrm{mmHg}$ to $19.3 \mathrm{mmHg}$. The only significant short-term complication in our study population was complete heart block, which developed after ASA in $44 \%$ of patients and necessitated insertion of a pacing implantable cardioverter-defibrillator prior to discharge.

Baseline serum levels of the biomarkers in the HCM patients and the healthy control cohort are presented in Table 3. The baseline serum concentration of all biomarkers, except for MMP-9, sICAM-1, and myeloperoxidase, was elevated in the ASA group compared with the controls. After alcohol infusion, serum markers of myocardial necrosis (TnI, CK-MB, and myoglobin) rose rapidly (Figure 1). The serum concentration of TnI rose over 800-fold, CK-MB by 70-fold, and myoglobin by 11 -fold $(P<0.002)$. Peak levels were evident for myoglobin at 8 hours, CK-MB at $8-16$ hours, and TnI at $16-24$ hours. Levels of BNP trended downward after 16 hours to levels significantly less than baseline at 48 hours $(P=0.03)$.

Among the inflammatory biomarkers, $\mathrm{C}$-reactive protein rose slowly to levels 3.6 -fold above baseline by 48 hours postASA $(P \leq 0.008)$. In contrast, levels of IL-6 gradually rose to 1.2 -fold above baseline at 48 hours $(P<0.0001)$, whereas
Table 3 Baseline serum biomarker levels in ASA patients and healthy controls

\begin{tabular}{|c|c|c|c|c|}
\hline $\begin{array}{l}\text { Serum } \\
\text { biomarkers }\end{array}$ & $\mathbf{n}$ & Mean & $\begin{array}{l}\text { Fold to } \\
\text { control }\end{array}$ & SD \\
\hline \multicolumn{5}{|c|}{ CK-MB (ng/mL) } \\
\hline Control & 107 & 2.16 & & 1.49 \\
\hline Baseline & 19 & 2.92 & 1.352 & 1.72 \\
\hline \multicolumn{5}{|c|}{ Myoglobin (ng/mL) } \\
\hline Control & 107 & 28.70 & & 14.90 \\
\hline Baseline & 19 & 33.76 & 1.176 & 20.03 \\
\hline \multicolumn{5}{|l|}{ IL-I $\beta$ (pg/mL) } \\
\hline Control & 103 & 34.42 & & 247.99 \\
\hline Baseline & 19 & 87.20 & 2.533 & 328.46 \\
\hline \multicolumn{5}{|l|}{ IL-6 (pg/mL) } \\
\hline Control & 103 & 34.23 & & 247.94 \\
\hline Baseline & 19 & 76.67 & 2.240 & $|29.7|$ \\
\hline \multicolumn{5}{|c|}{ TNF- $\alpha$ (pg/mL) } \\
\hline Control & 103 & 13.97 & & 62.17 \\
\hline Baseline & 19 & 53.65 & 3.840 & 109.45 \\
\hline \multicolumn{5}{|c|}{ MMP-9 (ng/mL) } \\
\hline Control & 103 & 300.22 & & 256.80 \\
\hline Baseline & 19 & 252.22 & 0.840 & 396.16 \\
\hline \multicolumn{5}{|l|}{ MPO (ng/mL) } \\
\hline Control & 103 & 161.89 & & 209.13 \\
\hline Baseline & 19 & 149.66 & 0.924 & 234.40 \\
\hline \multicolumn{5}{|c|}{ sICAM-I (ng/mL) } \\
\hline Control & 103 & 301.47 & & 334.70 \\
\hline Baseline & 19 & 230.47 & 0.764 & 123.96 \\
\hline \multicolumn{5}{|c|}{$\mathrm{sCD} 40 \mathrm{~L}(\mathrm{pg} / \mathrm{mL})$} \\
\hline Control & 103 & $6,367.57$ & & $12,620.40$ \\
\hline Baseline & 19 & $11,082.10$ & 1.740 & $7,000.96$ \\
\hline \multicolumn{5}{|l|}{ CRP (mg/L) } \\
\hline Control & 103 & 23.38 & & 46.05 \\
\hline Baseline & 19 & 35.78 & 1.530 & 59.02 \\
\hline \multicolumn{5}{|l|}{ BNP (pg/mL) } \\
\hline Control & 107 & 13.25 & & 10.31 \\
\hline Baseline & 19 & 326.05 & 24.608 & 308.28 \\
\hline \multicolumn{5}{|l|}{ Tnl (ng/mL) } \\
\hline Control & 106 & 0.02 & & 0.01 \\
\hline Baseline & 19 & 0.04 & 2.000 & 0.07 \\
\hline \multicolumn{5}{|l|}{ Adipo (ng/mL) } \\
\hline Control & 103 & $18,567.33$ & & $10,154.42$ \\
\hline Baseline & 19 & $24,440.00$ & 1.316 & II,599.38 \\
\hline
\end{tabular}

Abbreviations: ASA, alcohol septal ablation; CK-MB, creatine kinase MB; BNP, brain natriuretic peptide; CRP, C-reactive protein; IL, interleukin; TNF- $\alpha$, tumor necrosis factor-alpha; MPO, myeloperoxidase; MMP-9, matrix metalloproteinase-9; Adipo, adiponectin; sICAM-I, soluble intercellular adhesion molecule I; sCD40L, soluble CD40 ligand; Tnl, cardiac troponin l; SD, standard deviation.

IL- $1 \beta$ and TNF- $\alpha$ decreased during the measurement period $(P=0.03)$. Myeloperoxidase did not show any appreciable change after ASA (Figure 2).

MMP-9 displayed rapid kinetics, increasing 1.6-fold by 8 hours $(P=0.0002)$ and thereafter returned to baseline. Biomarkers associated with adhesion and atherosclerosis trended downward after ASA, with SCD40L demonstrating the greatest declines by 48 hours postprocedure $(P \leq 0.0003)$. 


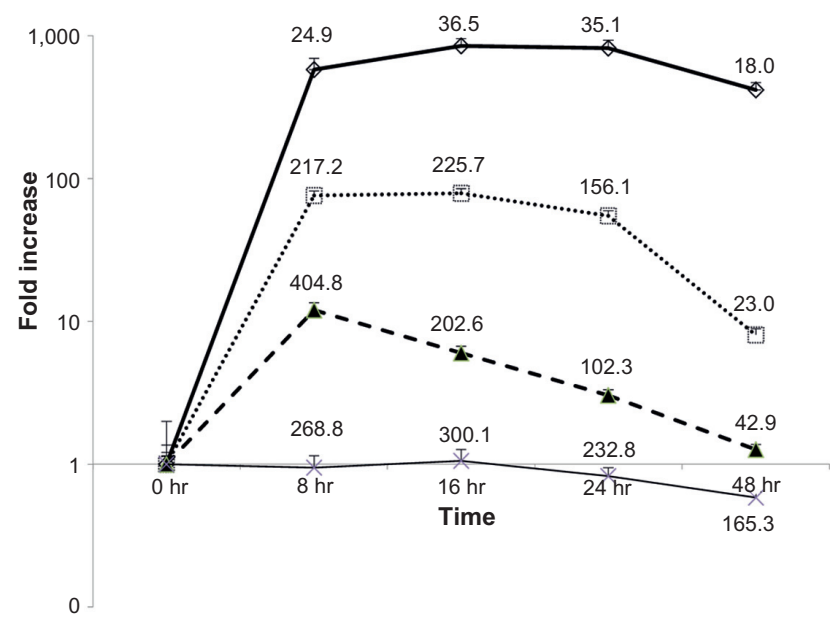

Figure I Temporal trend of serum biomarkers associated with myocardial damage due to alcohol septal ablation relative to baseline. The figure represents the "fold increase". The numbers above the symbols are concentration. Error bars are the mean standard error. Time points for cardiac troponin I ( $\mathrm{ng} / \mathrm{mL}$; diamond, $\diamond)$, creatine kinase MB band $(\mathrm{ng} / \mathrm{mL}$; square, $\square)$, and myoglobin $(\mathrm{ng} / \mathrm{mL}$; triangle, $\boldsymbol{\Lambda})$ were significantly different from baseline $(P<0.002)$ between 8 and 48 hours. Brain natriuretic peptide levels $(X ; p g /$ $\mathrm{mL})$ were significantly different from baseline at 48 hours $(P=0.03)$.

\section{Discussion}

This investigation sought to further characterize the physiological state that accompanies HCM and to correlate serum biomarker release with ASA-induced myocardial injury. Biomarkers associated with inflammation, atherosclerosis, thrombosis, myocardial strain, and myocardial damage were evaluated in serum. Our analyses resulted in three observations: concentrations of several serum biomarkers associated with the proinflammatory and prothrombotic state in atherosclerosis are elevated among patients with HCM when compared with healthy controls; ASA-induced changes in several serum biomarkers levels appear to reflect the degree of myocardial damage associated with the procedure; and some biomarkers that typically remain elevated in the days following spontaneous AMI fall rapidly following ASA. This may reflect infarct-related "improvement" in the left ventricular outflow tract gradient, a reduction in myocardial strain, or differences in the biological processes between ASA and AMI.

Notable in this study, we documented important differences in the concentration of several biomarkers among patients with HCM relative to controls. These findings are consistent with the idea that patients with HCM have altered an structural, inflammatory, and chemical signaling milieu. In this analysis, HCM patients were found to have higher levels of adiponectin, BNP, C-reactive protein, IL-1 $\beta$, IL-6, myoglobin, TnI, TNF- $\alpha$, and sCD40L than controls, who were negative for cardiovascular disease and cardiac symptoms and were assumed to have normal serum levels of these biomarkers. Conversely, MMP-9, myeloperoxidase, and sICAM-1 were significantly lower at baseline than controls. Intuitively, these findings support the idea that patients with HCM have dynamic outflow tract obstruction and significant amounts of septal hypertrophy that influence local tissue growth and left ventricular strain, and produce a proinflammatory environment. Similar studies have shown that when compared with age-matched and sex-matched controls, patients with HCM have elevated concentrations of biomarkers associated with endothelial dysfunction, and with thrombosis and platelet dysfunction. ${ }^{17,18}$

In this study, we characterize the release of biomarkers of thrombosis, inflammation, myocardial damage, and necrosis following ASA. We found that traditional biomarkers of myocardial damage, namely TnI, CK-MB, and myoglobin, are released in a temporal trend resembling that associated with spontaneous AMI, and could serve as a measure of infarct size in the setting of ASA. The kinetics of serum biomarker release in relation to the extent of myocardial damage associated with ASA have been studied previously. ${ }^{6,13,19}$ Hage et al demonstrated that serum levels of TnI, creatine kinase, and CK-MB correlated with the size of the ASA-induced infarct, whereas BNP did not. The mean infarct size in that study was $10 \%$ of the left ventricle or a mass of $19.1 \mathrm{~g} .{ }^{6}$ Although we did not specifically measure infarct size, our results mirror theirs, and our echocardiographic data at baseline and follow-up support the fact that our ASA cohort had a clinically successful procedure as measured by immediate improvements in left ventricular outflow tract gradients in close to $85 \%$ of our cohort.

One could hypothesize, based on the results of our two studies, that postprocedural levels of TnI or CK-MB could predict ASA-related infarct size and correlate with clinical improvement in this patient population. Other novel findings from this study include the fact that C-reactive protein and IL-6, two chemokines in the inflammatory cascade, rose throughout the entire postprocedural course, reflective of the immediate proinflammatory environment following ASA.

Other biomarkers appear to behave quite differently in the setting of ASA than in spontaneous AMI. In this analysis, BNP levels fell significantly following ASA. It is reasonable to speculate that an improvement in hemodynamics and a reduction in myocardial strain or myocardial thickness are related to the reduction in BNP observed in the current study. Similar findings were reported by Liebetrau et al, who observed an early increase in N-terminal of the prohormone brain natriuretic peptide (NT-Pro-BNP) levels in minutes following ASA, consistent with a response 

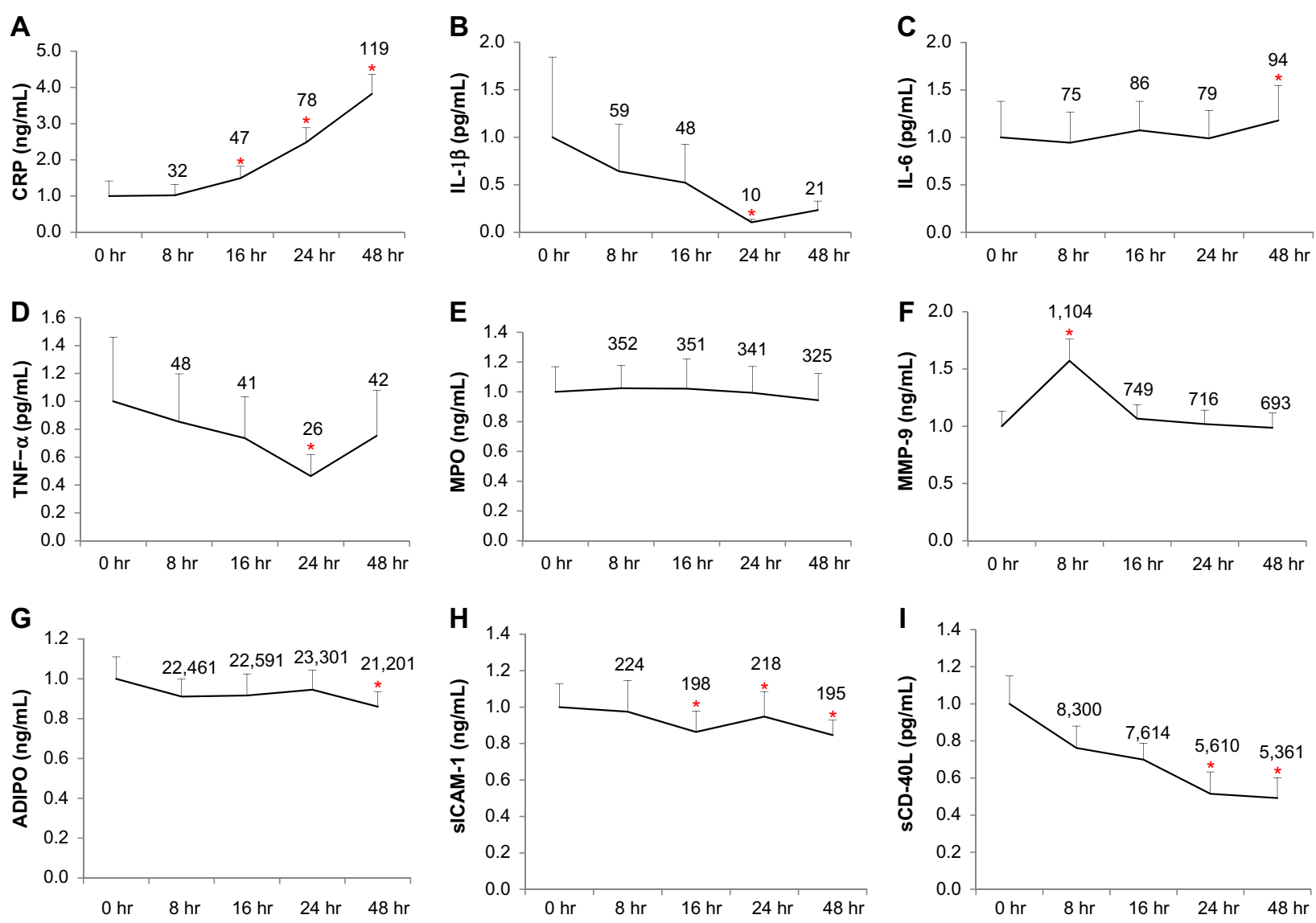

Figure 2 Cytokine biomarker kinetics following alcohol septal ablation.

Notes: Serum fold changes relative to baseline. (A) CRP, (B) IL-I $\beta$, (C) IL-6, (D) TNF- $\alpha$, (E) MPO, (F) MMP-9, (G) Adipo, (H) sICAM-I, and (I) sCD40L. Error bars are the mean standard error. Time points significantly different from baseline (as noted in Table 3$)(P<0.00 \mathrm{I})$ are indicated with an asterisk.

Abbreviations: CRP, C-reactive protein; IL, interleukin; TNF- $\alpha$, tumor necrosis factor-alpha; MPO, myeloperoxidase; MMP-9, matrix metalloproteinase-9; Adipo, adiponectin; sICAM-I, soluble cellular adhesion molecule I; sCD40L, soluble CD40 ligand.

to ischemia. At approximately 4 hours postprocedure, however, the median NT-Pro-BNP levels had started to decrease relative to baseline. ${ }^{19}$

IL-1 $\beta$, sCD40L, and TNF- $\alpha$ concentrations also decreased during the postprocedural course following ASA. All of these peptides are known to increase during and after an AMI. The declining sCD40L levels are particularly interesting as activated platelets are the source of this peptide in serum, and persistently elevated sCD40L levels are associated with adverse outcomes among patients with AMI. ${ }^{20}$ Each of these biomarkers were found at higher concentration in patients with HCM than in healthy controls at baseline, but fell during the observation period in contrast with the levels of traditional markers of myocardial injury such as TnI. It is reasonable to speculate that the reduction in the intercavitary gradient and the improvement in hemodynamics associated with ASA would have an effect on the inflammatory and thrombotic cascade that is distinct from that associated with a spontaneous MI. These findings, ie, the rise in markers of myocardial damage and the fall in BNP and sCD40L, suggest a role for biomarkers in determining the prognosis of patients following ASA. Further studies could correlate biomarker levels with clinical improvement, the subsequent need for a permanent pacing, or for the development of ventricular dysrhythmias.

Patients undergoing ASA experience chest pain, electrocardiographic changes, and myocardial necrosis. Studies with cardiac magnetic resonance imaging indicate the development of transmural infarctions in the majority of cases. ${ }^{21}$ These later findings also highlight the fact that there are significant differences in the pathophysiology of an AMI induced by the toxic effects of alcohol and one that arises from the rupture of atherosclerotic plaque. Specifically, ethanol is directly toxic to myocardial cells, resulting in death and presumably cell rupture with the release of intracellular enzymes such as TnI. The period of ischemia is defined and small, and unlikely to be associated with myocardial hemorrhage, as is found in AMI with prolonged ischemic times. Additionally, there is no reason to suspect the patients would experience recurrent waves of ischemia as is thought to occur in spontaneous AMI 
where the artery opens and closes and where microemboli are thought to inflict damage and platelet activation at sites distal to the arterial injury. In particular, the serum concentration of SCD40L may have been attenuated by the short ischemic time and intraprocedural use of heparin.

The biomarker profiles identified in this study are interesting, but should be interpreted with caution because of the obvious limitations in our study design, namely the small sample size. Another limitation includes the lack of a formal assessment of infarct size and subsequent correlation between biomarker levels and infarct size. On the other hand, the temporal trends in biomarker release demonstrated in our study mirror those of previous evaluations that performed assessments of ischemic time, suggesting that the findings have external validity. An additional limitation is that our control subjects were not matched demographically to our study cohorts. Finally, the serum concentrations of C-reactive protein, TNF- $\alpha$, sCD40L, IL-6, adiponectin, MMP-9, IL-1 $\beta$, myeloperoxidase, and sICAM-1 were determined using Luminex IS100-based multiplex kits. Thus, the conditions of the assay may not have been optimized for each individual biomarker.

\section{Conclusion and future perspectives}

Our findings support the premise that HCM, like atherosclerosis, is associated with a proinflammatory and prothrombotic state. When compared with healthy controls, patients with HCM were found to have elevated serum concentrations of biomarkers associated with inflammation, thrombosis, and endothelial proliferation. The temporal trends in the release of biomarkers of myocardial ischemia (TnI, CK-MB, myoglobin, and MMP-9) are similar to AMI, and likely reflect the size of the iatrogenic infarct. Other biomarkers, typically elevated in the setting of spontaneous AMI, fell during the measurement period, perhaps reflecting a reduction in intercavitary gradient or myocardial strain. Routine measurement of these biomarkers may prove to be prognostic following ASA, specifically the combination of serum troponin and BNP. Thus, these findings pave the way for larger studies in which biomarkers are used to investigate early biochemical events following the development of ischemia and the value of biomarkers for predicting long-term outcomes following ASA.

\section{Acknowledgments}

This study was supported by grants from the National Institute of Health (P20 RR020145 and M01-RR02602) and the University of Kentucky General Clinical Research Core, and was partially supported by a Clinical Translational Science Award (UL1RR033173). We thank Dawn G Dawson,
Justin R Kolasa, and Jason Stevens from the Department of Oral Health Practice, College of Dentistry, University of Kentucky, Lexington, KY, USA. Additionally, we thank the University of Kentucky Clinical Chemistry Laboratory.

\section{Disclosure}

The authors report no conflicts of interest in this work.

\section{References}

1. Maron BJ, Maron MS. Hypertrophic cardiomyopathy. Lancet. 2013;381(9862):242-255.

2. Kimmelstiel C. Role of percutaneous septal ablation in hypertrophic obstructive cardiomyopathy. Circulation. 2004;109(4):452-456.

3. Knight C. Nonsurgical septal reduction for hypertrophic obstructive cardiomyopathy: outcome in the first series of patients. Circulation. 1997;95(8):2075-2081.

4. Sigwart U. Non-surgical myocardial reduction for hypertrophic obstructive cardiomyopathy. Lancet. 1995;(346):211-214.

5. Valeti US, Nishimura RA, Holmes DR, et al. Comparison of surgical septal myectomy and alcohol septal ablation with cardiac magnetic resonance imaging in patients with hypertrophic obstructive cardiomyopathy. J Am Coll Cardiol. 2007;49(3):350-357.

6. Hage FG, Aqel R, Aljaroudi W, et al. Correlation between serum cardiac markers and myocardial infarct size quantified by myocardial perfusion imaging in patients with hypertrophic cardiomyopathy after alcohol septal ablation. Am J Cardiol. 2010;105(2):261-266.

7. Adams J. Cardiac troponin I. A marker with high specificity for cardiac injury. Circulation. 1993;88(1):101-106.

8. Wu A. Comparison of myoglobin, creatine kinase-MB, and cardiac troponin I for diagnosis of acute myocardial infarction. Ann Clin Lab Sci. 1996;26(4):291-300.

9. Madsen LH, Lund T, Grieg Z, et al. Cardiac troponin I degradation in serum of patients with hypertrophic obstructive cardiomyopathy undergoing percutaneous septal ablation. Cardiology. 2009;114(3):167-173.

10. Liebetrau C, Mollmann H, Nef H, et al. Release kinetics of cardiac biomarkers in patients undergoing transcoronary ablation of septal hypertrophy. Clin Chem. 2012;58(6):1049-1054.

11. Bradham WS, Gunasinghe H, Holder JR, et al. Release of matrix metalloproteinases following alcohol septal ablation in hypertrophic obstructive cardiomyopathy. J Am Coll Cardiol. 2002;40(12):2165-2173.

12. Sakamoto T, Mizuno Y, Ogawa H, Yoshimura M, Kugiyama K, Yasue H. B-type natriuretic peptide after percutaneous transluminal septal myocardial ablation. Int J Cardiol. 2002;83(2):151-158.

13. Liebetrau C, Nef HM, Dorr O, et al. Release kinetics of early ischaemic biomarkers in a clinical model of acute myocardial infarction. Heart. 2014;100(8):652-657.

14. Lewis GD, Wei R, Liu E, et al. Metabolite profiling of blood from individuals undergoing planned myocardial infarction reveals early markers of myocardial injury. J Clin Invest. 2008;118(10):3503-3512.

15. Foley JD 3rd, Sneed JD, Steinhubl SR, et al. Salivary biomarkers associated with myocardial necrosis: results from an alcohol septal ablation model. Oral Surg Oral Med Oral Pathol Oral Radiol. 2012;114(5):616-623.

16. Foley JD 3rd, Sneed JD, Steinhubl SR, et al. Oral fluids that detect cardiovascular disease biomarkers. Oral Surg Oral Med Oral Pathol Oral Radiol. 2012;114(2):207-214.

17. Dimitrow PP, Undas A, Bober M, Tracz W, Dubiel JS. Plasma biomarkers of endothelial dysfunction in patients with hypertrophic cardiomyopathy. Pharmacol Rep. 2007;59(6):715-720.

18. Dimitrow PP, Undas A, Bober M, Tracz W, Dubiel JS. Obstructive hypertrophic cardiomyopathy is associated with enhanced thrombin generation and platelet activation. Heart. 2008;94(6):e21. 
19. Liebetrau C, Gaede L, Dorr O, et al. Release kinetics of N-terminal pro-B-type natriuretic peptide in a clinical model of acute myocardial infarction. Clin Chim Acta. 2014;429:34-37.

20. Pamukcu B, Lip GY, Snezhitskiy V, Shantsila E. The CD40-CD40L system in cardiovascular disease. Ann Med. 2011;43(5):331-340.
21. Mather AN, Fairbairn TA, Artis NJ, Greenwood JP, Plein S. Relationship of cardiac biomarkers and reversible and irreversible myocardial injury following acute myocardial infarction as determined by cardiovascular magnetic resonance. Int J Cardiol. 2013;166(2):458-464.

\section{Publish your work in this journal}

Current Biomarker Findings is an international, peer-reviewed, open access journal publishing original research, reports, reviews and commentaries on all areas of biomarker research. The manuscript management system is completely online and includes a very quick and fair

\section{Dovepress}

peer-review system. Visit http://www.dovepress.com/testimonials.php to read real quotes from published authors. 